ARTICLE

Received 10 Nov 2013 | Accepted 9 Apr 2014 | Published 12 May 2014 DOl: 10.1038/ncomms4841

\title{
Observation of quantum-tunnelling-modulated spin texture in ultrathin topological insulator $\mathrm{Bi}_{2} \mathrm{Se}_{3}$ films
}

Madhab Neupane ${ }^{1}$, Anthony Richardella ${ }^{2}$, Jaime Sánchez-Barriga ${ }^{3}$, SuYang Xu' ${ }^{1}$, Nasser Alidoust ${ }^{1}$,lya Belopolski ${ }^{1}$, Chang Liu', Guang Bian', Duming Zhang ${ }^{2}$, Dmitry Marchenko 3,4 , Andrei Varykhalov ${ }^{3}$, Oliver Rader ${ }^{3}$, Mats Leandersson ${ }^{5}$, Thiagarajan Balasubramaniann, ${ }^{5}$ Tay-Rong Chang ${ }^{6}$, Horng-Tay Jeng ${ }^{6,7}$, Susmita Basak ${ }^{8}$, Hsin Lin ${ }^{9}$, Arun Bansil ${ }^{8}$, Nitin Samarth ${ }^{2} \&$ M. Zahid Hasan ${ }^{1,10}$

Understanding the spin-texture behaviour of boundary modes in ultrathin topological insulator films is critically essential for the design and fabrication of functional nanodevices. Here, by using spin-resolved photoemission spectroscopy with p-polarized light in topological insulator $\mathrm{Bi}_{2} \mathrm{Se}_{3}$ thin films, we report tunnelling-dependent evolution of spin configuration in topological insulator thin films across the metal-to-insulator transition. We report a systematic binding energy- and wavevector-dependent spin polarization for the topological surface electrons in the ultrathin gapped-Dirac-cone limit. The polarization decreases significantly with enhanced tunnelling realized systematically in thin insulating films, whereas magnitude of the polarization saturates to the bulk limit faster at larger wavevectors in thicker metallic films. We present a theoretical model that captures this delicate relationship between quantum tunnelling and Fermi surface spin polarization. Our high-resolution spin-based spectroscopic results suggest that the polarization current can be tuned to zero in thin insulating films forming the basis for a future spin-switch nanodevice.

\footnotetext{
${ }^{1}$ Joseph Henry Laboratory, Department of Physics, Princeton University, Princeton, New Jersey 08544, USA. ${ }^{2}$ Department of Physics, The Pennsylvania State University, University Park, Pennsylvania 16802, USA. ${ }^{3}$ Helmholtz-Zentrum Berlin für Materialien und Energie, Elektronenspeicherring BESSY II,

Albert-Einstein-Strasse 15, D-12489 Berlin, Germany. ${ }^{4}$ Physikalische und Theoretische Chemie, Freie Universität Berlin, Takustraße 3, 14195 Berlin, Germany. ${ }^{5}$ MAX-lab, PO Box 118, S-22100 Lund, Sweden. ${ }^{6}$ Department of Physics, National Tsing Hua University, Hsinchu 30013, Taiwan. ${ }^{7}$ Institute of Physics, Academia Sinica, Taipei 11529, Taiwan. ${ }^{8}$ Department of Physics, Northeastern University, Boston, Massachusetts 02115, USA. ${ }^{9}$ Graphene Research Centre, Department of Physics, National University of Singapore, Singapore 117542, Singapore. ${ }^{10}$ Princeton Center for Complex Materials, Princeton University, Princeton, New Jersey 08544, USA. Correspondence and requests for materials should be addressed to M.Z.H. (email: mzhasan@princeton.edu).
} 
A three-dimensional (3D) topological insulator (TI) is a non-trivial phase of matter that acts as an electrical insulator in the bulk but can conduct a spin-polarized current on the surface ${ }^{1-19}$. These topological surface states are characterized by a Dirac-cone-like energy-momentum dispersion relation. The novel electronic structure of TIs can be manipulated to realize various novel quantum phenomena such as spingalvanic effects, dissipationless spin currents or neutral halffermions for quantum information storage devices ${ }^{20-25}$. The magnitude and wavevector dependence of the spin polarization of electrons and holes are among the most important key ingredients in considerations for the design of any functional device. However, such developments have been limited because of the residual bulk conductance in currently available materials, which overwhelms the surface contribution. In addition, scattering from the extrinsic bulk states leads to the reduction of spin polarization of the surface states. One promising route to minimize bulk conductance and thus improve effective spin polarization is to work with ultrathin films where the surface-tovolume ratio is significantly enhanced ${ }^{26,27}$ and surface current can potentially dominate. On the other hand, in this limit the desired spin polarization of the surface states is kinematically reduced near the metal-to-insulator transition in the ultrathin films where the spin behaviour is not known to this date $10,11,15,18,19$.

To date, no systematic spin-sensitive spectroscopic experimental study has been reported in the ultrathin limit across the metal-to-insulator transition, despite the direct relevance of spins in ultrathin film limits for nanodevice fabrication as well as the potential discovery of novel topological phenomena. Studying the spin polarization in the ultrathin limit is further important to experimentally demonstrate the theoretically predicted tunable Berry's phase in TI thin films ${ }^{28}$. Systematic mapping of the surface spin texture in energy, momentum space and its thickness dependence is essential to understand and interpret many transport experiments on thin film TIs that are of core interest in the current TI research. We report a systematic spin-resolved, angle-resolved photoemission spectroscopy (SR-ARPES) and spin-integrated ARPES measurements on ultrathin $\mathrm{Bi}_{2} \mathrm{Se}_{3}$ thin films for the first time. Our measurements reveal that the spin polarization is large for larger wavevectors, and the polarization magnitude increases with reduced tunnelling, and its magnitude saturates to the bulk limit at a faster rate at large electron momenta.

We observe strongly binding energy- and wavevector-dependent spin polarization for the topological surface electrons in the ultrathin gapped-Dirac-cone limit, which experimentally shows that the Dirac gap opening and the thickness-dependent topological phase transition are a result of the quantum tunnelling between the two oppositely spin-textured topological surface states. These unique spin features of ultrathin films, evidently distinct from the 3D TI, open up new possibilities for devices not possible with bulk TIs.

\section{Results}

Sample characterization. Spectroscopic measurements were performed on large ultrathin $\mathrm{Bi}_{2} \mathrm{Se}_{3}$ films prepared using the Molecular Beam Epitaxy (MBE) method on GaAs(111)A substrates (Fig. 1a). Each crystal layer of $\mathrm{Bi}_{2} \mathrm{Se}_{3}$ is constituted of five atomic layer, namely $\mathrm{Se}-\mathrm{Bi}-\mathrm{Se}-\mathrm{Bi}-\mathrm{Se}$, which is called quintpule layer (QL) with the thickness of $\sim 1 \mathrm{~nm}$ (ref. 6). Our MBE films grow in a self-organized quintuple layer by quintuple-layer mode, and high-quality atomically smooth films can be obtained with the desired thickness (also see Supplementary Figs 1-7 and Supplementary Notes 1-3). A compositional layout of the film used in our measurements is shown in Fig. 1b. To protect the surface from contamination, about $40-\mathrm{nm}$ Se capping is used on the top of ultrathin $\mathrm{Bi}_{2} \mathrm{Se}_{3}$ films. To expose the surface, the films were transferred into the ARPES chamber and heated to $250^{\circ} \mathrm{C}$ at pressures lower than $1 \times 10^{-9}$ torr about an hour, which blows off the Se-capping layer. Figure 1c shows the ARPES core level spectroscopy measurement of the ultrathin film before and after decapping of the Se layer. Before decapping, only Se peaks are visible while both Se and Bi peaks are observed after the decapping process, which proves that the Se capping works well in the ultrathin film TI system. Thin films are characterized with atomic force microscopy (see Fig. 1d) and show that the root mean square (r.m.s.) surface roughness on these films is in the order of $\sim 0.2 \mathrm{~nm}$, which confirms the high quality of the films used in our measurements. The transport measurements of the Se-capped ultrathin films result in carrier concentration, mobility and resistivity in the order of $1 \times 10^{19} \mathrm{~cm}^{-3}, 1,270 \mathrm{~cm}^{2} \mathrm{~V}^{-1} \mathrm{~S}^{-1}$ and $0.30 \mathrm{mOhm} \mathrm{cm}$, respectively (also see Supplementary Fig. 1 and Supplementary Note 1).

Geometry of the spectroscopic measurements. We used p-polarized light for our ARPES measurements. The photons approach the sample surface with angle of incident $(\theta) 45$ degree with the sample normal and our samples are aligned along $\bar{\Gamma}-\overline{\mathrm{K}}$ momentum space cut (Fig. 2a) for spin-ARPES measurements. The surface wavevector-dependent spin polarization is obtained using a Mott polarimeter (Fig. 2a), which measures two orthogonal spin components of a photoemitted electron ${ }^{29,30}$. In the polarimeter, a gold foil was used as a scattering source to generate an asymmetry of high energy photoelectrons into different divergent spin states (see refs 29,30 for details). Each orthogonal spin-polarization component is selected by the orientation of a scattering plane defined by the incident beam direction of the photoelectron on the gold foil and the orientation of two electron detectors mounted on each side of the foil. For this experiment, the detector configuration was set in a way that the two spin components correspond to the in-plane and out-of-plane directions of the (111) plane of the sample.

Thickness- and wavevector-dependent spin polarization. We present high-resolution spin-integrated ARPES data and corresponding energy distribution curves (EDCs) along the highsymmetry line $\bar{\Gamma}-\overline{\mathrm{K}}$ for $1 \mathrm{QL}, 3 \mathrm{QL}, 4 \mathrm{QL}, 6 \mathrm{QL}$ and $7 \mathrm{QL} \mathrm{Bi}_{2} \mathrm{Se}_{3}$ films in Fig. 2c,d. As long as the thickness of the film is comparable to the decay length of the surface states into the bulk, there is a spatial overlap between the top and bottom surface states resulting in an energy gap at the time-reversal invariant point ( $\bar{\Gamma}$ point $)$. As expected theoretically ${ }^{26,27}$, the energy gap decreases and eventually vanishes for sufficiently thick films, corresponding to the transition from a two-dimensional (2D) gapped system (insulator) to a $3 \mathrm{D}$ gapless system (metal) ${ }^{10,11}$. In particular, the gapless dispersion relation observed in the $7 \mathrm{QL}$ film from ARPES measurement indicates that this thickness is above the quantum tunnelling limit. On the basis of experimental observations, we present an illustration of the spin configuration for 3QL (insulator; see Supplementary Figs 2 and 3 and Supplementary Note 2) and 7QL (metal) ultrathin $\mathrm{Bi}_{2} \mathrm{Se}_{3}$ films in Fig. 2b. It is important to note that a wide range of electronic structures have been reported in ultrathin TI films grown by MBE depending on the nature of the substrates used ${ }^{10,11,18}$. Different substrates result in different potential jumps from the substrate (the bottom surface of the ultrathin film) to the vacuum (the top surface of the film). When the potential is large, the Dirac point energy of the bottom surface is offset with respect to that of the top surface. Such Dirac point energy offset is observed to cause 
a
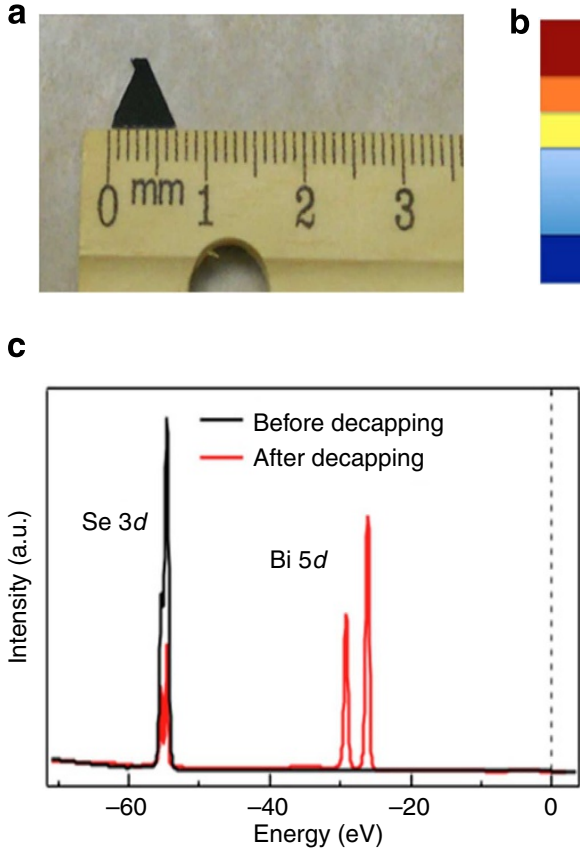

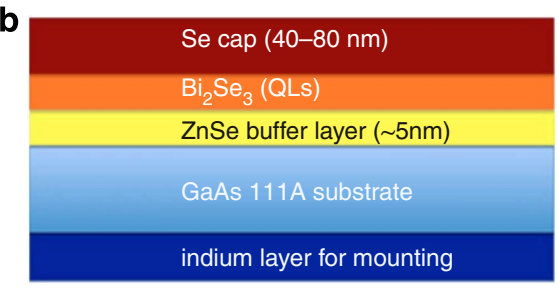

d

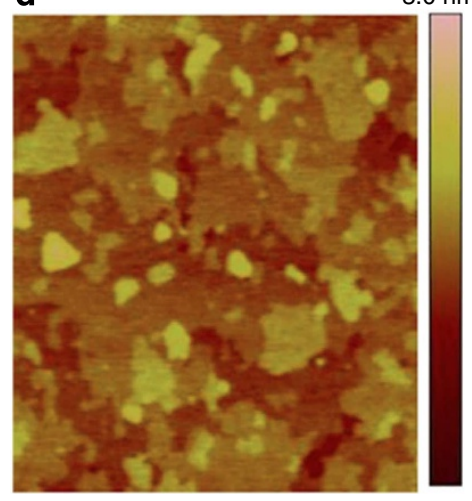

Figure 1 | Characterization of $\mathbf{M B E}$ grown ultrathin films of $\mathbf{B i}_{\mathbf{2}} \mathbf{S e}_{\mathbf{3}}$. (a) Photograph of a representative thin film sample used in SR-ARPES measurements. (b) Sample layout of ultrathin $\mathrm{Bi}_{2} \mathrm{Se}_{3}$ film grown by MBE. (c) Core level spectroscopy measurements on ultrathin MBE film before and after the decapping procedure. (d) Atomic force microscopy image of ultrathin $\mathrm{Bi}_{2} \mathrm{Se}_{3}$ film. The size of the image is $1 \mu \mathrm{m} \times 1 \mu \mathrm{m}$ and height is indicated by the colour bar on the right.

sizeable Rashba-type splitting of surface states as reported in ultrathin $\mathrm{Bi}_{2} \mathrm{Se}_{3}$ films grown on double-layer grapheneterminated $6 \mathrm{H}-\mathrm{SiC}(0001)$ substrate ${ }^{10}$ and Bi-terminated Si(11) $-(7 \times 7)($ ref. 18$)$ substrate by MBE, respectively. On the other hand, no observable Rashba-type splitting because of substrate potential is reported in the $\mathrm{Bi}_{2} \mathrm{Se}_{3}$ grown on $\mathrm{Si}(111) \beta(3)^{1 / 2} \times(3)^{1 / 2}-\mathrm{Bi}$ substrate (in ref. 11), $\alpha-\mathrm{Al}_{2} \mathrm{O}_{3}$ (sapphire) (0001) substrate (in ref. 19) and our current work $\mathrm{GaAs}(111) \mathrm{A}$.

We investigate the degree of spin polarization of the films as a function of electron wavevector for films of various thicknesses. To illustrate the wavevector-dependent spin polarization, $3 \mathrm{QL}$ film is chosen (see Supplementary Figs 2 and 3 and Supplementary Note 2 for details). The SR-ARPES data for the wavevectors, $\sim 0.05$ and $0.1 \AA^{-1}$ are presented in Fig. 3. For each wavevector selected, we present a spin-resolved EDC, which shows the relative intensity of photoelectrons with up and down spin polarizations (Fig. 3a,c). For each such plot we associate a net spin polarization with the surface state of a given wavevector (Fig. 3b,d) and for 3QL film $\sim 25$ and $15 \%$ are estimated at momenta $\sim 0.1$ and $\sim 0.05 \AA^{-1}$, respectively. From these plots it is clearly observed that the net spin polarization decreases for smaller wavevectors (locations $k \sim 0.05 \AA^{-1}$ in Fig. 3d). The decrease can be understood as the presence of a tunnelling gap in the ultrathin limit that effectively prevents the partner-switching behaviour expected in the gapless topological surface states' system $^{27}$. The tunnelling gap for ultrathin films can be seen in the data (Fig. 2c). The reduction in the spin polarization and the existence of a tunnelling gap in the data suggest that the righthanded contribution of the bottom surface to the spin polarization must increase to effectively cancel the left-handed helical spin texture of the top surface upon approaching smaller momentum values near $\bar{\Gamma}$.

Figure 3a shows the spin-resolved EDCs for $3 \mathrm{QL}, 4 \mathrm{QL}, 6 \mathrm{QL}$ and $60 \mathrm{QL}$ films while the corresponding net polarization is shown in Fig. $3 \mathrm{~b}$ at $k=0.1 \AA^{-1}$. Analogous measurements are shown in Fig. 3c,d at $k=0.05 \AA^{-1}$ for $1 \mathrm{QL}, 3 \mathrm{QL}, 4 \mathrm{QL}, 6 \mathrm{QL}$ and $60 \mathrm{QL}$ films. For thinner films, such as $3 \mathrm{QL}$, there is a naturally larger contribution from the bottom surface (because of tunnelling between top and bottom surfaces). This leads to a larger variation in spin polarization as a function of wavevector. For thicker films, this tunnelling contribution decreases and the surface spin polarization becomes more uniform with varying wavevector. For instance, in the $60 \mathrm{QL}$ film, no measurable change in spin polarization is observed for the variation in wavevector magnitude. Our data suggest that the magnitude of polarization tends to reach the bulk limit faster at larger wavevectors. Experimental results are summarized in Fig. 4a. Data further suggest that the relative contribution from the top surface systematically increases with film thickness.

Model calculation. First-principles theoretical modelling of the spin-polarization behaviour in thin films is presented in Fig. 4b,c. In the calculations, symmetric slabs are used to simulate the thickness of films. While a gapless spin-polarized Dirac-cone is seen on the surface of a semi-infinite crystal of the TI $\mathrm{Bi}_{2} \mathrm{Se}_{3}$, a gap is found to open at the Dirac point for thin films because of a finite tunnelling amplitude between the two sides of the slab surface in our calculations. The tunnelling amplitude increases as the thickness decreases, causing the gap to increase and spin polarization to decrease in the gap region. In the calculation we also consider the electron attenuation length $(\lambda)$ because of the scattering processes since only electrons near the surface are able to reach the top of the surface and escape into the vacuum as in the measurement condition. Indeed, the spin polarization obtained by ARPES reflects the spin texture of the states associated with top surface rather than the bottom surface. The calculated spin expectation value for the electrons that can escape from the sample is $\langle S\rangle_{\text {atom }} \times \exp \left(-d_{\text {atom }} / \lambda\right)$, where $d_{\text {atom }}$ is the 


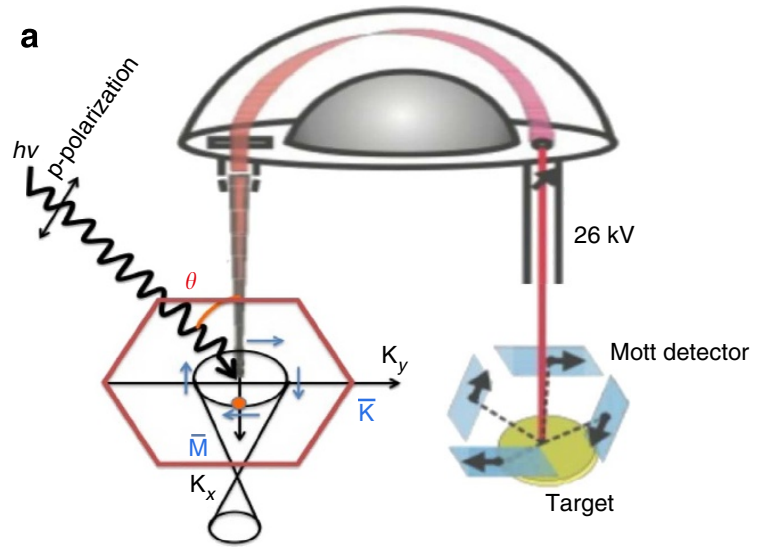

b
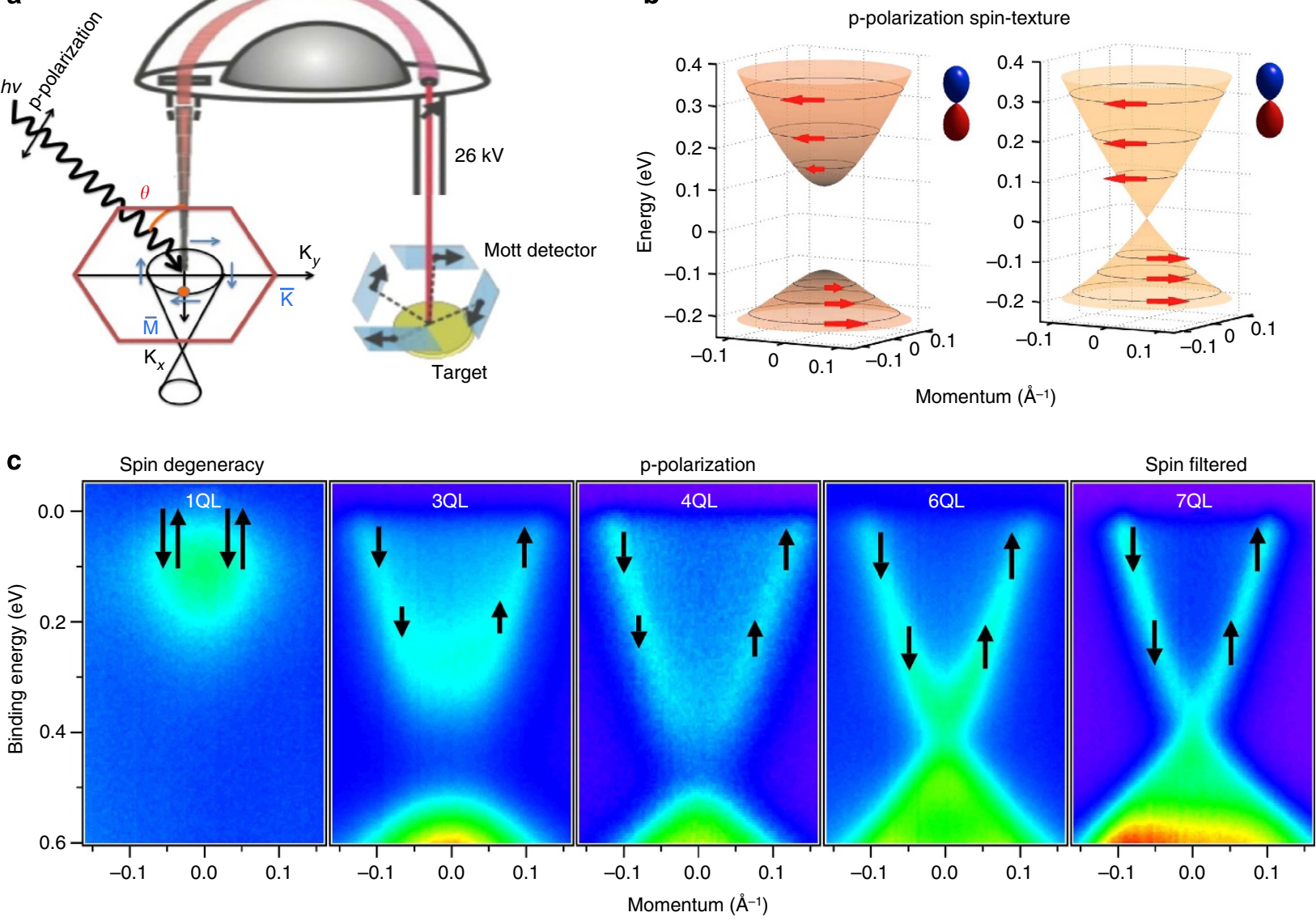

d

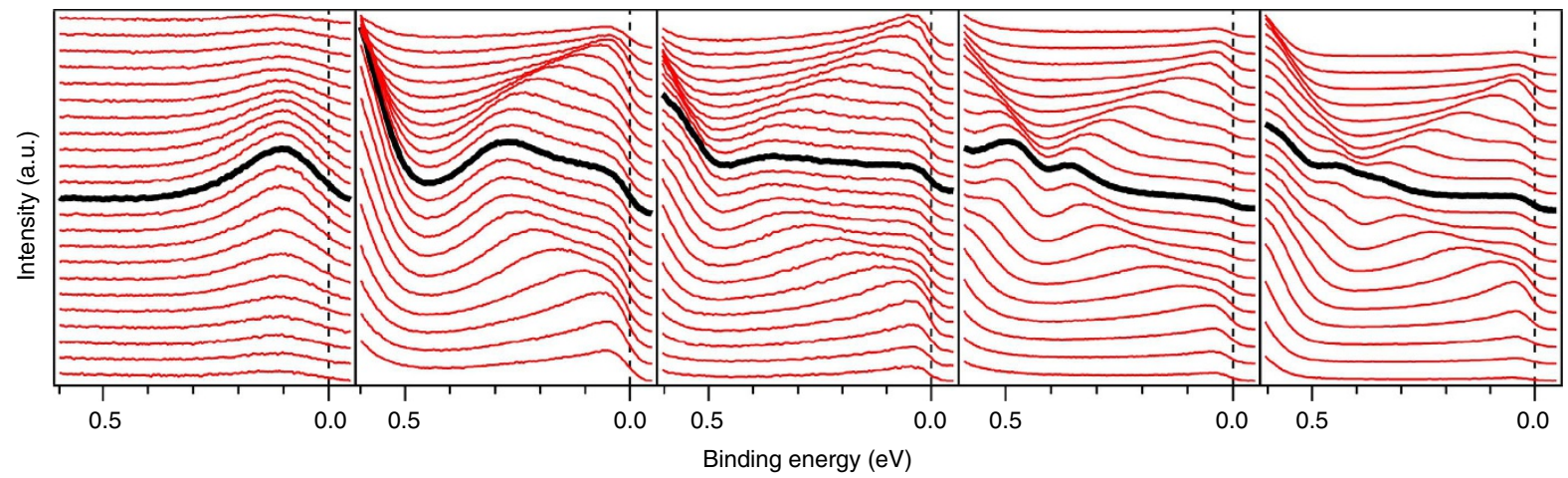

Figure 2 | Spin-texture versus quantum tunnelling in ultrathin $\mathbf{B i}_{\mathbf{2}} \mathbf{S e}_{\mathbf{3}}$. (a) Experimental geometry used in our measurements. (b) Visualization of the contrasting spin configurations in $3 \mathrm{QL}$ (insulator) and $7 \mathrm{QL}$ (metal) thin films. The dumbbell signs indicate that the current experimental geometry mainly probes the $p_{z}$ orbitals of $\mathrm{Bi}$ and $\mathrm{Se}$. (c) High-resolution ARPES measurements on ultrathin films of $\mathrm{Bi}_{2} \mathrm{Se}_{3}$ : $E-k$ band dispersion images for $1 \mathrm{QL}$, $3 \mathrm{QL}, 4 \mathrm{QL}, 6 \mathrm{QL}$ and $7 \mathrm{QL}$ of $\mathrm{Bi}_{2} \mathrm{Se}_{3}$ films taken near the $\bar{\Gamma}$ point along $\bar{\Gamma}-\overline{\mathrm{K}}$ high-symmetry direction. The spin configuration is noted on the plots.

These spectra are measured with photon energy of $60 \mathrm{eV}$. (d) The corresponding EDCs. The EDC through the $\bar{\Gamma}$ point (solid black curve) is highlighted.

distance of an atom to the top surface, and the $\langle S\rangle_{\text {atom }}$ is the spin expectation value for each atom. The contribution from each atom is weighted by $\exp \left(-d_{\text {atom }} / \lambda\right)$, which reduces the contribution from the bottom layer. Figure 4 b,c shows the calculated results with $\lambda=8 \AA$, which agrees excellently with our experimental observation (see Supplementary Methods for details related to calculations).

\section{Discussion}

It is important to note that the maximum spin polarization observed in the bulk limit is only $\sim 40 \%$ (Fig. $4 \mathrm{c}$ ), whereas the original ideal theoretical limit is that of nearly $100 \%$ without considering any specific material system ${ }^{31}$. In real TI materials, the strong spin-orbit interaction entangles the spin and orbital momenta of different atomic types, resulting in the reduction of spin polarization ${ }^{32}$. Specifically, the low-energy states in $\mathrm{Bi}_{2} \mathrm{Se}_{3}$ arise from $p$-orbitals of $\mathrm{Bi}\left(6 p^{3}\right)$ and Se $\left(4 p^{4}\right)$, mostly $p_{z}$ levels of $\mathrm{Bi}$ and $\mathrm{Se}^{6,12}$. The dominance of the $p_{z}$ orbitals in the topological surface states is further suggested by our circular dichroism measurements (see Supplementary Fig. 4; Supplementary Note 2 and Supplementary Methods). The spin-orbit coupling mixes spin and orbital angular momenta while preserving the total 


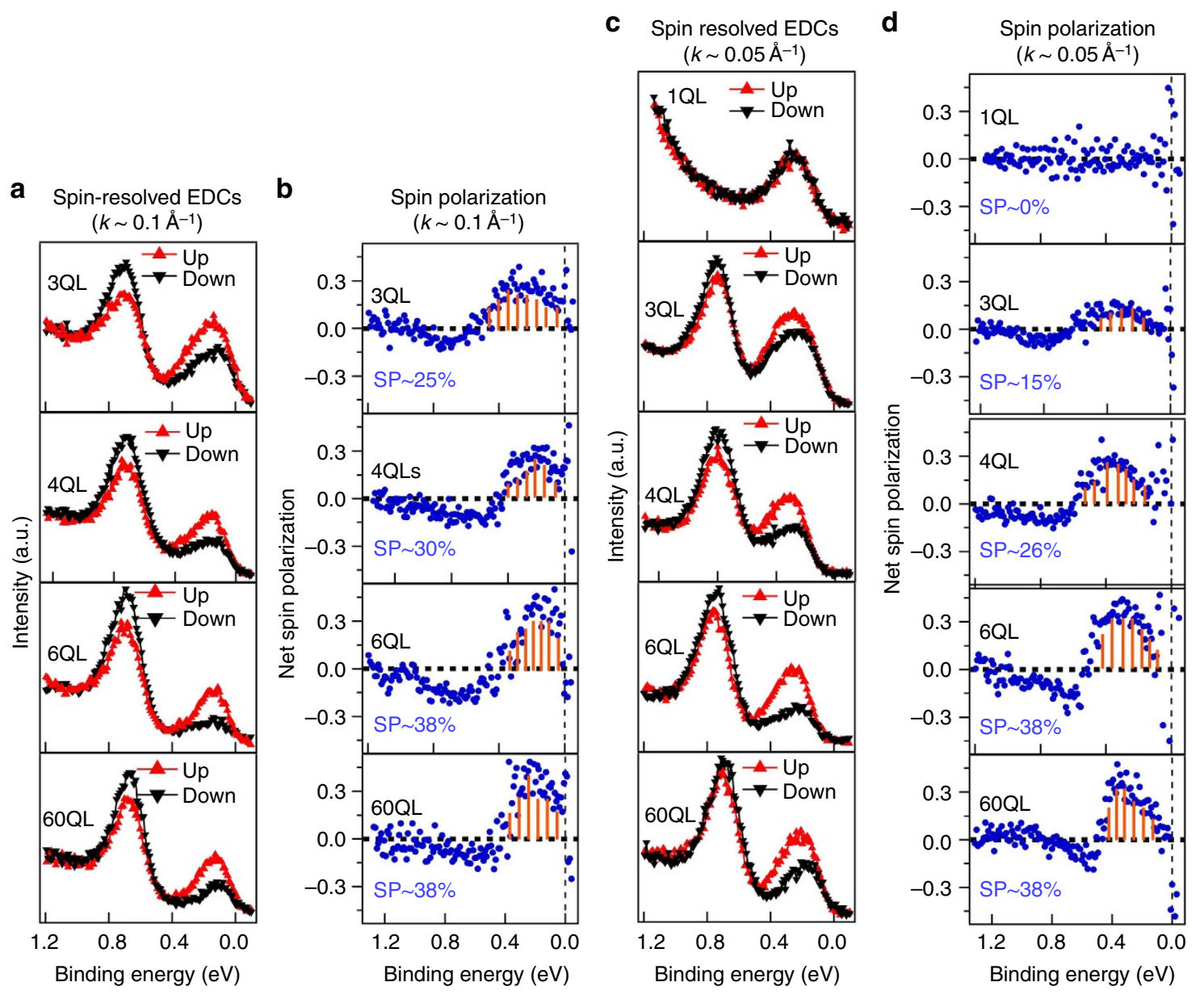

Figure 3 | Thickness-dependent quantum tunnelling and evolution of spin configuration. (a) Spin-resolved EDCs and (b) net tangential spin polarizations for $3 \mathrm{QL}, 4 \mathrm{QL}, 6 \mathrm{QL}$ and $60 \mathrm{QL}$ ultrathin $\mathrm{Bi}_{2} \mathrm{Se}_{3}$ films at $k \sim 0.1 \AA^{-1}$. c,d same as a,b for $1 \mathrm{QL}, 3 \mathrm{QL}, 4 \mathrm{QL}, 6 \mathrm{QL}$ and $60 \mathrm{QL}$ ultrathin $\mathrm{Bi}_{2} \mathrm{Se} \mathrm{e}_{3}$ films at $k \sim 0.05 \AA^{-1}$. The red (black) curves show tangentially up (down) spin-resolved EDCs. The magnitude of each net spin polarization is also noted in $(\mathbf{b}, \mathbf{d})$. The vertical red lines in $\mathbf{b}, \mathbf{d}$ are guides to the eye, indicating a non-zero area under the spin polarization curve. SR-ARPES data were collected using photon energy of $60 \mathrm{eV}$. We note that $1 \mathrm{QL}$ is equivalent to $\sim 1 \mathrm{~nm}$.

angular momentum 6,12 . The hybridization of orbitals in $\mathrm{Bi}$ and Se together with the entanglement of their spins contribute to the reduction of net spin polarization in real materials. Moreover, under the experimental geometry used in our measurement with p-polarized light (which is most sensitive to $p_{z}$ orbitals and most reflective of initial ground state of the wavefunction), the penetration depth of the ARPES experiment (three to five atomic layers maximum), the experimental observation of spin polarization is well agreed with recent theoretical calculations $^{33-35}$.

Most importantly, our systematic spin spectroscopy results suggest that ultrathin films can serve as the basis for making qualitatively new devices, not possible with much-studied conventional 3D bulk TIs, despite the reduction of the polarization magnitude. The energy-momentum space spin texture revealed in our study provides critical knowledge to design and interpret devices based on ultrathin films. For spintronics applications, our results suggest that ultrathin TI films can be used to fabricate new types of nanodevices because of the novel spin configurations and their systematic modulations possible in the ultrathin limit. One such potential application implied by our spectroscopic results is that of a polarization current switch. Spin spectroscopic results suggest that it should be possible to control the polarization magnitude by varying a gate voltage (Fig. $4 \mathrm{~d}, \mathrm{e}$ ) across a high-quality insulating thin film (see supplementary Fig. 5 and Supplementary Note 3). Such electrostatic gating would be similar to the in situ chemical gating by K-deposition and $\mathrm{NO}_{2}$ adsorption shown in Supplementary Figs 6 and 7, where the surface state structure does not change appreciably, while the Fermi energy is moved from regions of high polarization to low polarization (see Supplementary Note 3). This effect, which cannot be readily realized in the highly polarized states of conventional 3D bulk TIs, allows ultrathin TI films to serve as the basis for functional nanodevices, which can encode electrical signals in varying spin polarization magnitude or forms the physical basis for a spin switch, among many other new application possibilities suggested by our observations of the fundamental spin modulation behaviour in ultrathin films.

\section{Methods}

Sample growth and transport measurements. Ultrathin $\mathrm{Bi}_{2} \mathrm{Se}_{3}$ films used for this study were synthesized by MBE on a GaAs(111)A substrate with a ZnSe buffer layer. Details of sample preparation are described elsewhere ${ }^{36,37}$. To protect the surface from oxidation, a thick Se-capping layer was deposited on the $\mathrm{Bi}_{2} \mathrm{Se}_{3}$ thin film immediately after growth. To achieve the clean $\mathrm{Bi}_{2} \mathrm{Se}_{3}$ surface required for photoemission measurements, thin films were heated up inside the ARPES chamber to $250^{\circ} \mathrm{C}$ under vacuum better than $1 \times 10^{-9}$ torr to remove the Secapping layer on top of ultrathin $\mathrm{Bi}_{2} \mathrm{Se}_{3}$. Hall bars fabricated from the Se-capped samples had carrier concentrations in the $1-2 \times 10^{19} \mathrm{~cm}^{-3}$ range. Transport measurements were carried out on Hall bars of the 5 and 6 QL thick films with the 


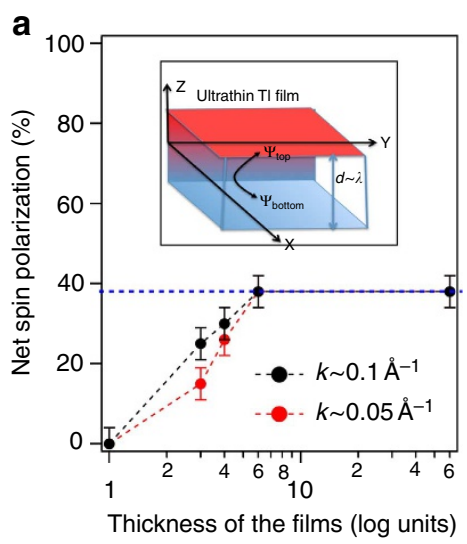

d
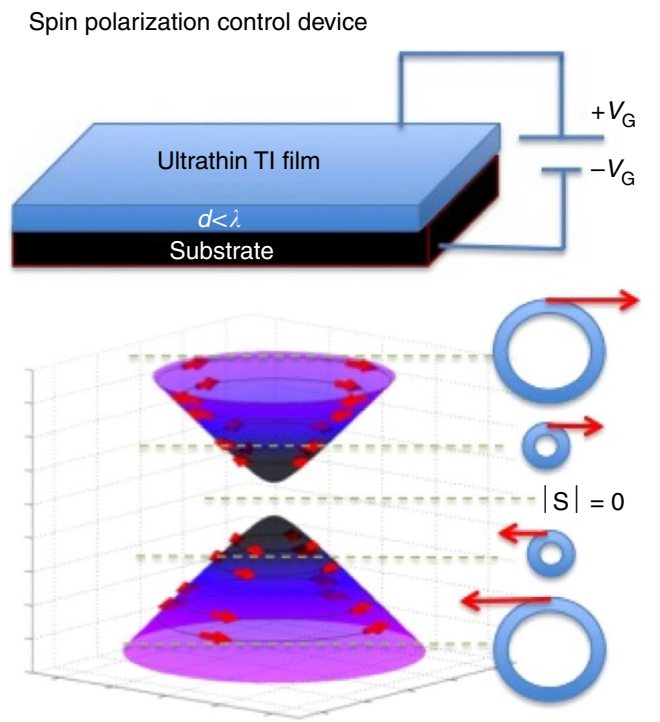

b

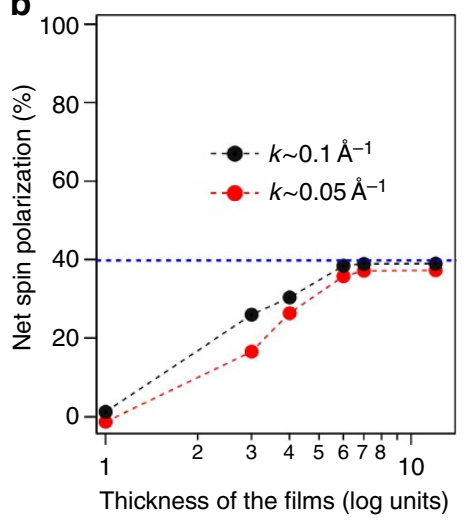

C

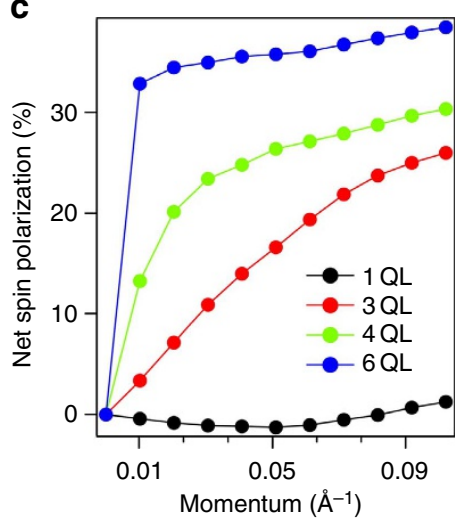

e

Insulating TI film

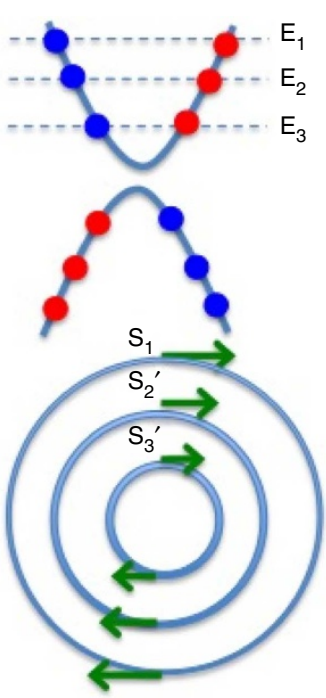

Metallic TI film and bulk 3D TI

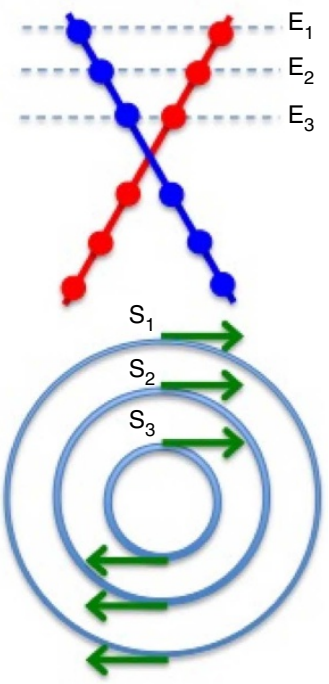

Figure 4 | Experimental versus theoretical spin polarization and texture. (a) Experimentally observed net spin polarization as a function of thin film thickness for $k \sim 0.05$ and $0.1 \AA^{-1}$. The inset shows a schematic view of quantum tunnelling in ultrathin TI films. (b) Calculation results of spin polarization versus film thickness at two momentum points $k \sim 0.05$ and $0.1 \AA^{-1}$. (c) Results of a calculation of the net spin polarization as a function of wavevector for ultrathin films of thickness 1QL, 3QL, 4QL and 6QL. Dashed lines in a,b and solid lines in $\mathbf{c}$ between the dots serve as guides to the eye. Error bars in a represent experimental uncertainties in determining the spin polarization. Schematic of (d) a gate controlled spin polarization current switch device and (e) momentum-dependent spin configuration in ultrathin (insulating) film and thicker (metallic) film.

Se-capping layer in place by standard photolithography and dry etching and were measured at $4.2 \mathrm{~K}$.

High-resolution ARPES measurements. High-resolution spin-integrated ARPES measurements were performed with $29-64 \mathrm{eV}$ photon energy on beamlines 10.0.1 and 12.0.1 at the Advanced Light Source in Lawrence Berkeley National Laboratory. Both endstations were equipped with a Scienta hemispherical electron analyser (see VG Scienta manufacturer website (http://www.vgscienta.com/) for instrument specifications). The typical energy and momentum resolutions were $15 \mathrm{meV}$ and $1 \%$ of the surface Brillouin zone, respectively, for spin-integrated measurements.

Spin-resolved ARPES measurements. Spin-resolved ARPES measurements were performed at the UE112-PGM1 beamline at Bessy II in Berlin, Germany, and the I3 beamline at Maxlab in Lund, Sweden, using classical Mott detectors and photon energies 55-60 and 8-20 eV, respectively. The typical energy and momentum resolutions were $100 \mathrm{meV}$ and $3 \%$ of the surface Brillouin zone for spin-resolved measurements. All the SR spectra presented are measured in BESSY II unless it is specified.

Theoretical calculations. The first-principles calculations for spin polarizations of ultrathin films are based on the generalized gradient approximation ${ }^{38}$ using the full-potential projected augmented wave method ${ }^{39,40}$ as implemented in the VASP package ${ }^{41}$. The $1 \mathrm{QL}, 3 \mathrm{QL}, 4 \mathrm{QL}, 6 \mathrm{QL}, 7 \mathrm{QL}$ and $12 \mathrm{QL}$ slab models with a vacuum thickness larger than $10 \AA$ are used in this work. The electronic structure calculations were performed over $11 \times 11 \times 1$ Monkhorst-Pack k-mesh with the spin-orbit coupling included self-consistently.

\section{References}

1. Hasan, M. Z. \& Kane, C. L. Colloquium: topological insulators. Rev. Mod. Phys. 82, 3045-3067 (2010).

2. Moore, J. E. The birth of topological insulators. Nature 464, 194-198 (2010).

3. Qi, X.-L. \& Zhang, S.-C. Topological insulators and superconductors. Rev. Mod. Phys. 83, 1057-1110 (2011).

4. Fu, L. \& Kane, C. L. Topological insulators with inversion symmetry. Phys. Rev B 76, 045302 (2007).

5. Hsieh, D. et al. A topological Dirac insulator in a quantum spin Hall phase. Nature 452, 970-974 (2008).

6. Xia, Y. et al. Observation of a large-gap topological-insulator class with a single Dirac cone on the surface. Nat. Phys. 5, 398-402 (2009).

7. Chen, Y.-L. et al. Massive Dirac Fermion on the surface of a magnetically doped topological insulator. Science 329, 659-662 (2010).

8. Xu, S.-Y. et al. Observation of a topological crystalline insulator phase and topological phase transition in $\mathrm{Pb}_{1-x} \mathrm{Sn}_{x}$ Te. Nat. Commun. 3, 1192 (2012).

9. Neupane, M. et al. Surface electronic structure of the topological Kondo-insulator candidate correlated electron system $\mathrm{SmB}_{6}$. Nat. Commun. 4, 2991 (2013). 
10. Zhang, Y. et al. Crossover of the three-dimensional topological insulator $\mathrm{Bi}_{2} \mathrm{Se}_{3}$ to the two-dimensional limit. Nat. Phys. 6, 584-588 (2010).

11. Sakamoto, Y. et al. Spectroscopic evidence of a topological quantum phase transition in ultrathin $\mathrm{Bi}_{2} \mathrm{Se}_{3}$ films. Phys. Rev. B 81, 165432 (2010).

12. Zhang, $\mathrm{H}$. et al. Topological insulators in $\mathrm{Bi}_{2} \mathrm{Se}_{3}, \mathrm{Bi}_{2} \mathrm{Te}_{3}$ and $\mathrm{Sb}_{2} \mathrm{Te}_{3}$ with a single Dirac cone on the surface. Nat. Phys. 5, 438-442 (2009).

13. Hsieh, D. et al. A tunable topological insulator in the spin helical Dirac transport regime. Nature 460, 1101-1105 (2009).

14. Liu, C.-X. et al. Oscillatory crossover from two-dimensional to threedimensional topological insulators. Phys. Rev. B 81, 041307(R) (2010).

15. Jiang, Y. et al. Landau quantization and the thickness limit of topological insulator thin films of $\mathrm{Sb}_{2} \mathrm{Te}_{3}$. Phys. Rev. Lett. 108, 016401 (2012).

16. Taskin, A. A. et al. Manifestation of topological protection in transport properties of epitaxial $\mathrm{Bi}_{2} \mathrm{Se}_{3}$ thin films. Phys. Rev. Lett. 109, 066803 (2012).

17. Neupane, M. et al. Topological surface states and Dirac point tuning in ternary topological insulators. Phys. Rev. B 85, 235406 (2012).

18. Berntsen, M. H. et al. Direct observation of decoupled Dirac states at the interface between topological and normal insulators. Phys. Rev. B 88, 195132 (2013).

19. Chang, C.-Z. et al. Growth of topological insulator $\mathrm{Bi}_{2} \mathrm{Se}_{3}$ on insulating substrate. Spin 01, 21-25 (2011).

20. Garate, I. \& Franz, M. Inverse spin-galvanic effect in the interface between a topological insulator and a ferromagnet. Phys. Rev. Lett. 104, 146802 (2010).

21. Qi, X.-L. et al. Inducing a magnetic monopole with topological surface states. Science 323, 1184-1187 (2009).

22. Essin, A., Moore, J. E. \& Vanderbilt, D. Magnetoelectric polarizability and axion electrodynamics in crystalline insulators. Phys. Rev. Lett. 102, 146805 (2009).

23. Yu, R. et al. Quantized anomalous Hall effect in magnetic topological insulators. Science 329, 61-64 (2010).

24. Linder, J. et al. Unconventional superconductivity on a topological insulator Phys. Rev. Lett. 104, 067001 (2010).

25. Fu, L. \& Kane, C. L. Probing neutral Majorana fermion edge modes with charge transport. Phys. Rev. Lett. 102, 216403 (2009).

26. Linder, J., Yokoyama, T. \& Sudbo, A. Anomalous finite size effects on surface states in the topological insulator $\mathrm{Bi}_{2} \mathrm{Se}_{3}$. Phys. Rev. B 80, 205401 (2009).

27. Lu, H.-Z. et al. Massive Dirac fermions and spin physics in an ultrathin film of topological insulator. Phys. Rev. B 81, 115407 (2010).

28. Lu, H.-Z. et al. Competition between weak localization and antilocalization in topological surface states. Phys. Rev. Lett. 107, 076801 (2011).

29. Berntsen, M. H. A spin- and angle-resolving photoelectron spectrometer. Rev. Sci. Instrum. 81, 035104 (2010).

30. Dil, J. H. Spin and angle resolved photoemission on non-magnetic lowdimensional systems. J. Phys. Condens. Matter. 21, 403001 (2009).

31. Kane, C. L. \& Mele, E. J. $Z_{2}$ Topological order and the quantum spin Hall effect. Phys. Rev. Lett. 95, 146802 (2005).

32. Yazyev, O. V., Moore, J. E. \& Louie, S. G. Spin polarization and transport of surface states in the topological Insulators $\mathrm{Bi}_{2} \mathrm{Se}_{3}$ and $\mathrm{Bi}_{2} \mathrm{Te}_{3}$ from first principles. Phys. Rev. Lett. 105, 266806 (2010).

33. Zhu, -H. Z. et al. Layer-by-layer entangled spin-orbital texture of the topological surface state in $\mathrm{Bi}_{2} \mathrm{Se}_{3}$. Phys. Rev. Lett. 110, 216401 (2013).

34. Park, S. R. et al. Chiral orbital-angular momentum in the surface states of $\mathrm{Bi}_{2} \mathrm{Se}_{3}$. Phys. Rev. Lett. 108, 046805 (2012).

35. Sánchez-Barriga, J. et al. Photoemission of $\mathrm{Bi}_{2} \mathrm{Se}_{3}$ with circularly polarized light: probe of spin polarization or means for spin manipulation? Phys. Rev. X 4, 011046 (2014).

36. Xu, S.-Y. et al. Hedgehog spin texture and Berry's phase tuning in a magnetic topological insulator. Nat. Phys. 8, 616-622 (2012).
37. Zhang, D. et al. Interplay between ferromagnetism, surface states, and quantum corrections in a magnetically doped topological insulator. Phys. Rev. B 86, 205127 (2012).

38. Perdew, J. P., Burke, K. \& Ernzerhof, M. Generalized gradient approximation made simple. Phys. Rev. Lett. 77, 3865 (1996).

39. Blöchl, P. E. Projector augmented-wave method. Phys. Rev. B 50, 17953 (1994).

40. Kresse, G. \& Joubert, J. From ultrasoft pseudopotentials to the projector augmented-wave method. Phys. Rev. B 59, 1758 (1999).

41. Kress, G. \& Hafner, J. Ab initio molecular dynamics for open-shell transition metals. Phys. Rev. B 48, 13115 (1993).

\section{Acknowledgements}

Sample growth and ARPES characterization are supported by US DARPA (N66001-111-4110). The work at the Princeton University and Princeton-led synchrotron X-raybased measurements and the related theory at the Northeastern University are supported by the Office of Basic Energy Science, US Department of Energy (grants DE-FG-0205ER462000, AC03-76SF00098 and DE-FG02-07ER46352). M.Z.H. acknowledges visiting-scientist support from the Lawrence Berkeley National Laboratory and additional support from the A.P. Sloan Foundation. The spin-resolved and spin-integrated photoemission measurements using synchrotron X-ray facilities are supported by the Swedish Research Council, the Knut and Alice Wallenberg Foundation, the German Federal Ministry of Education and Research, and the Basic Energy Sciences of the US Department of Energy. Theoretical computations are supported by the US Department of Energy (DE-FG02-07ER46352 and AC03-76SF00098) as well as the National Science Council and Academia Sinica in Taiwan, and benefited from the allocation of supercomputer time at NERSC and Northeastern University's Advanced Scientific Computation Center. H.L. acknowledges the Singapore National Research Foundation for the support under NRF Award No. NRF-NRFF2013-03. T.R.C. and H.T.J. are supported by the National Science Council, Taiwan. H.T.J. also thanks NCHC, CINC-NTU and NCTS, Taiwan, for technical support. We also thank S.-K. Mo and A. Fedorov for beamline assistance on spin-integrated photoemission measurements (supported by DE-FG0205ER46200) at the Lawrence Berkeley National Laboratory (The synchrotron facility is supported by the US DOE).

\section{Author contributions}

M.N., J.S.-B., and S.X. performed the experiments with assistance from N.A., I.B., C.L. G.B., D.M., A.V., O.R., M.L., T.B. and M.Z.H.; A.R., D.Z. and N.S. provided thin film MBE samples and performed sample characterization; M.N. and M.Z.H. performed data analysis, figure planning and draft preparation. T.-R.C., H.-T.J., S.B., H.L. and A.B. carried out calculations; M.Z.H. was responsible for the conception and the overall direction, planning and integration among different research units.

\section{Additional information}

Supplementary Information accompanies this paper at http://www.nature.com/ naturecommunications

Competing financial interests: The authors declare no competing financial interests

Reprints and permission information is available online at http://npg.nature.com/ reprintsandpermissions/

How to cite this article: Neupane, M. et al. Observation of quantum-tunnellingmodulated spin texture in ultrathin topological insulator $\mathrm{Bi}_{2} \mathrm{Se}_{3}$ films. Nat. Commun 5:3841 doi: 10.1038/ncomms4841 (2014). 